Catalysis by Methyltrioxorhenium(VII). Reduction of Hydronium Ions by Europium(II) and

\title{
Reduction of Perchlorate Ions by Europium(II) and Chromium(II)
}

Yang Cai and James H. Espenson

\section{Supporting Information}

\section{Contents}

Table S-1. Kinetic data for the formation of L410 by MTO with $\mathrm{Eu}^{2+}$.

Table S-2. Kinetic data for hydrogen evolution by MTO and $\mathrm{Eu}^{2+}$.

Table S-3. Kinetic data for hydrogen evolution by MTO and $\mathrm{Eu}^{2+}$ with variations of acid concentration.

Table S-4. Pseudo-first-order rate constant $\mathrm{k}_{\mathrm{obs}}$ for hydrogen evolution with varying ionic strength

Table S-5. Quantitative measurements of chloride ion concentration for perchlorate ion reduction.

Table S-6. Kinetic data for perchlorate ion reduction

Table S-7. Rate constants for perchlorate ion reduction by transition metal complexes

Figure S-1. Calibration curve for hydrogen evolution.

Figure S-2. Spectrophotometric titrations of MTO with $\mathrm{Eu}^{2+}$.

Figure S-3. Absorbance change showing that $\mathrm{Eu}^{2+}$ is rapidly oxidized by MTO.

Figure S-4. Plot of $\mathrm{k}_{\psi}$ against the concentration of MTO for the $k_{1}$ reaction.

Figure S-5. Plot of $\mathrm{k}_{\mathrm{obs}}$ for eq 7 against the concentration of $\mathrm{Eu}^{2+}$

Figure S-6. Showing that the pseudo-first-order rate constant $\mathrm{k}_{\mathrm{obs}}$ remains independent of $\left[\mathrm{Eu}^{2+}\right]$.

Figure S-7. Repetitive scans of UV-vis spectra representing the decrease of $\mathrm{Eu}^{2+}$ for perchlorate reduction at $1 \mathrm{~s}$ intervals.

Figure S-8. Plot showing the linear variation of the pseudo-zero-order rate constant $\mathrm{k}_{\mathrm{obs}}$ with the concentration of $[\mathrm{Re}]_{\mathrm{T}}$.

Figure S-9. Plot showing the first-order dependence upon $\left[\mathrm{ClO}_{4}^{-}\right]$for perchlorate reduction

Figure S-10. Variations of $\mathrm{k}_{\mathrm{obs}} /\left[\mathrm{ClO}_{4}^{-}\right]$with $[\mathrm{Re}]_{\mathrm{T}}$ for perchlorate ion reduction.

Figure S-11. Effect of the order of addition on perchlorate reduction.

Figure S-12. Deliberate introduction of a trace of $\mathrm{O}_{2}$ greatly exaggerates the curvature during the MTO-catalyzed reduction of $\mathrm{ClO}_{4}^{-}$by $\mathrm{Eu}^{2+}$. 
Table S-1. Kinetic data for the formation of L410 by MTO with excess acidic Eu ${ }^{2+}$ solutions. Conditions: $\left[\mathrm{H}^{+}\right]=0.6$ $\mathrm{M}, \mu=0.62 \mathrm{M}$.

\begin{tabular}{lll}
\hline$\left[\mathrm{Eu}^{2+}\right] / 10^{-3} \mathrm{M}$ & {$[\mathrm{MTO}] / 10^{-3} \mathrm{M}$} & $\mathrm{k}_{\mathrm{obs}} / \mathrm{s}^{-1}$ \\
\hline 9.76 & 0.20 & 0.616 \\
5.08 & 0.20 & 0.315 \\
4.87 & 0.20 & 0.299 \\
3.81 & 0.20 & 0.244 \\
3.53 & 0.20 & 0.196 \\
3.25 & 0.20 & 0.201 \\
3.25 & 0.30 & 0.193 \\
3.25 & 0.26 & 0.198 \\
3.25 & 0.23 & 0.193 \\
3.25 & 0.20 & $0.197^{a}$ \\
2.83 & 0.20 & 0.153 \\
2.53 & 0.20 & 0.153 \\
2.19 & 0.20 & 0.118 \\
2.12 & 0.20 & 0.118 \\
\hline$a .\left[\mathrm{H}^{+}\right]=0.2 \mathrm{M}$ & &
\end{tabular}

Table S-2. Kinetic data for hydrogen evolution by MTO and $\mathrm{Eu}^{2+}$. Conditions: $\left[\mathrm{H}^{+}\right]=0.22 \mathrm{M}, \mu \sim 0.3 \mathrm{M}$ at $25^{\circ} \mathrm{C}$.

\begin{tabular}{lll}
\hline$\left[\mathrm{Eu}^{2+}\right] / 10^{-3} \mathrm{M}$ & $\mathrm{MTO} / 10^{-3} \mathrm{M}$ & $\mathrm{k}_{\mathrm{obs}} / 10^{-3} \mathrm{~s}^{-1}$ \\
\hline 12.8 & 0.193 & 2.07 \\
12.4 & 0.394 & 4.42 \\
12.4 & 0.320 & 3.48 \\
12.3 & 0.497 & 5.85 \\
9.9 & 0.320 & 3.54 \\
8.77 & 0.320 & 3.42 \\
8.03 & 0.320 & 2.88 \\
5.85 & 0.320 & 3.49 \\
5.07 & 0.320 & 3.47 \\
3.2 & 0.320 & 3.62 \\
\hline
\end{tabular}

Table S-3. Kinetic data for hydrogen evolution by $\mathrm{MTO}$ and $\mathrm{Eu}^{2+}$ with variations of acid concentration. Conditions: $[\mathrm{MTO}]=0.21 \mathrm{mM}, \mu=0.33 \mathrm{M}$ at $25^{\circ} \mathrm{C}$.

\begin{tabular}{lll}
\hline$\left[\mathrm{H}^{+}\right] / \mathrm{M}$ & {$\left[\mathrm{Eu}^{2+}\right] / 10^{-3} \mathrm{M}$} & $\mathrm{k}_{\mathrm{obs}} / 10^{-3} \mathrm{~s}^{-1}$ \\
\hline 0.114 & 6.94 & 4.80 \\
0.127 & 6.41 & 4.39 \\
0.150 & 6.6 & 3.58 \\
0.170 & 8.42 & 3.13 \\
0.194 & 6.94 & 2.55 \\
0.222 & 6.84 & 2.31 \\
0.246 & 6.6 & 2.25 \\
0.298 & 6.94 & 1.69 \\
\hline
\end{tabular}


Table S-4. Pseudo-first-order rate constant $\mathrm{k}_{\mathrm{obs}}$ for hydrogen evolution with varying ionic strength at $0.10 \mathrm{M} \mathrm{H}^{+}$

\begin{tabular}{lllll}
\hline$\mu / \mathrm{M}$ & $F_{\mu}$ & {$\left[\mathrm{Eu}^{2+}\right] / 10^{-3} \mathrm{M}$} & {$[\mathrm{MTO}] / 10^{-3} \mathrm{M}$} & $\mathrm{k}_{\text {obs }} / 10^{-3} \mathrm{~s}^{-1}$ \\
\hline 0.118 & 0.2557 & 5.66 & 0.354 & 13.0 \\
0.118 & 0.2557 & 5.66 & 0.354 & 13.1 \\
0.121 & 0.2581 & 5.00 & 0.216 & 7.72 \\
0.142 & 0.2737 & 5.08 & 0.216 & 7.68 \\
0.161 & 0.2864 & 5.00 & 0.216 & 7.37 \\
0.19 & 0.3036 & 5.66 & 0.354 & 11.2 \\
0.246 & 0.3315 & 5.08 & 0.216 & 6.73 \\
0.297 & 0.3527 & 5.00 & 0.216 & 5.93 \\
\hline
\end{tabular}

${ }^{\mathrm{a}} F_{\mu}=\mu^{1 / 2} /\left(1+\mu^{1 / 2}\right)$

Table S-5. Quantitative measurements of chloride ion concentration for the reaction of perchlorate ion reduction.

\begin{tabular}{llll}
\hline$\left[\mathrm{Eu}^{2+}\left(\right.\right.$ or $\left.\left.\mathrm{Cr}^{2+}\right)\right]$ & {$[\mathrm{MTO}]$} & {$\left[\mathrm{Cl}^{-}\right]_{\text {measured }}$} & {$\left[\mathrm{Eu}^{2+}\left(\right.\right.$ or $\left.\left.\mathrm{Cr}^{2+}\right)\right] /\left[\mathrm{Cl}^{-}\right]$} \\
$10^{-3} \mathrm{M}$ & $10^{-3} \mathrm{M}$ & $10^{-3} \mathrm{M}$ & \\
\hline 8.00 & 0.20 & 0.958 & 8.4 \\
4.00 & 0.08 & 0.479 & 8.4 \\
3.00 & 0.08 & 0.406 & 7.4 \\
2.00 & 0.08 & 0.228 & 8.8 \\
1.00 & 0.08 & 0.136 & 7.4 \\
\hline
\end{tabular}

Table S-6. Kinetic data for perchlorate ion reduction with $\left[\mathrm{H}^{+}\right]=0.55 \mathrm{M}$ at $\mu=0.8 \mathrm{M}$.

\begin{tabular}{llll}
\hline$\left[\mathrm{ClO}_{4}{ }^{-}\right] / \mathrm{M}$ & {$[\mathrm{MTO}] / 10^{-4} \mathrm{M}$} & {$\left[\mathrm{Eu}^{2+}\right] / 10^{-3} \mathrm{M}$} & $\mathrm{k}_{\text {obs }} / 10^{-4} \mathrm{M} \mathrm{s}^{-1}$ \\
\hline 0.050 & 0.965 & 1.55 & 2.11 \\
0.050 & 0.965 & 1.24 & 2.15 \\
0.050 & 0.965 & 1.86 & 1.93 \\
0.030 & 1.00 & 1.55 & 1.11 \\
0.050 & 1.00 & 1.55 & 2.00 \\
0.070 & 1.00 & 1.55 & 2.97 \\
0.100 & 1.00 & 1.55 & 4.80 \\
0.120 & 1.00 & 1.55 & 5.38 \\
0.050 & 0.595 & 1.23 & 1.21 \\
0.050 & 0.760 & 1.23 & 1.64 \\
0.050 & 1.21 & 1.23 & 2.54 \\
0.050 & 1.58 & 1.23 & 3.38 \\
0.050 & 1.97 & 1.23 & 4.49 \\
0.040 & 0.98 & 1.55 & 1.53 \\
0.100 & 0.98 & 1.55 & 4.50 \\
\hline
\end{tabular}

Table S-7. Rate constants for perchlorate ion reduction by transition metal complexes.

\begin{tabular}{|l|l|l|}
\hline Reducing agent & $\mathrm{T} /{ }^{\circ} \mathrm{C}$ & $k / \mathrm{L} \mathrm{mol}^{-1} \mathrm{~s}^{-1}$ \\
\hline $\mathrm{V}_{\mathrm{aq}}{ }^{3+}$ & 50 & $2.8 \times 10^{-6}$ \\
\hline $\mathrm{Ru}\left(\mathrm{H}_{2} \mathrm{O}\right)_{6}{ }^{2+}$ & 25 & $3.2 \times 10^{-3}$ \\
\hline $\mathrm{Ti}_{\mathrm{aq}}{ }^{3+}$ & 50 & $1.5 \times 10^{-3}$ \\
\hline $\mathrm{Ru}\left(\mathrm{NH}_{3}\right)_{5}\left(\mathrm{H}_{2} \mathrm{O}\right)^{2+}$ & 25 & $2.7 \times 10^{-2}$ \\
\hline $\mathrm{Ru}\left(\mathrm{NH}_{3}\right)_{6}{ }^{2+}$ & 25 & $3.0 \times 10^{-4}$ \\
\hline $\mathrm{V}_{\mathrm{aq}}{ }^{2+}$ & 50 & $2.2 \times 10^{-5}$ \\
\hline $\mathrm{Eu}_{\mathrm{aq}}{ }^{2+}$ & 28 & $\sim 10^{-8}$ \\
\hline $\mathrm{Cr}_{\mathrm{aq}}{ }^{2+}$ & $\sim 25$ & no reaction \\
\hline
\end{tabular}



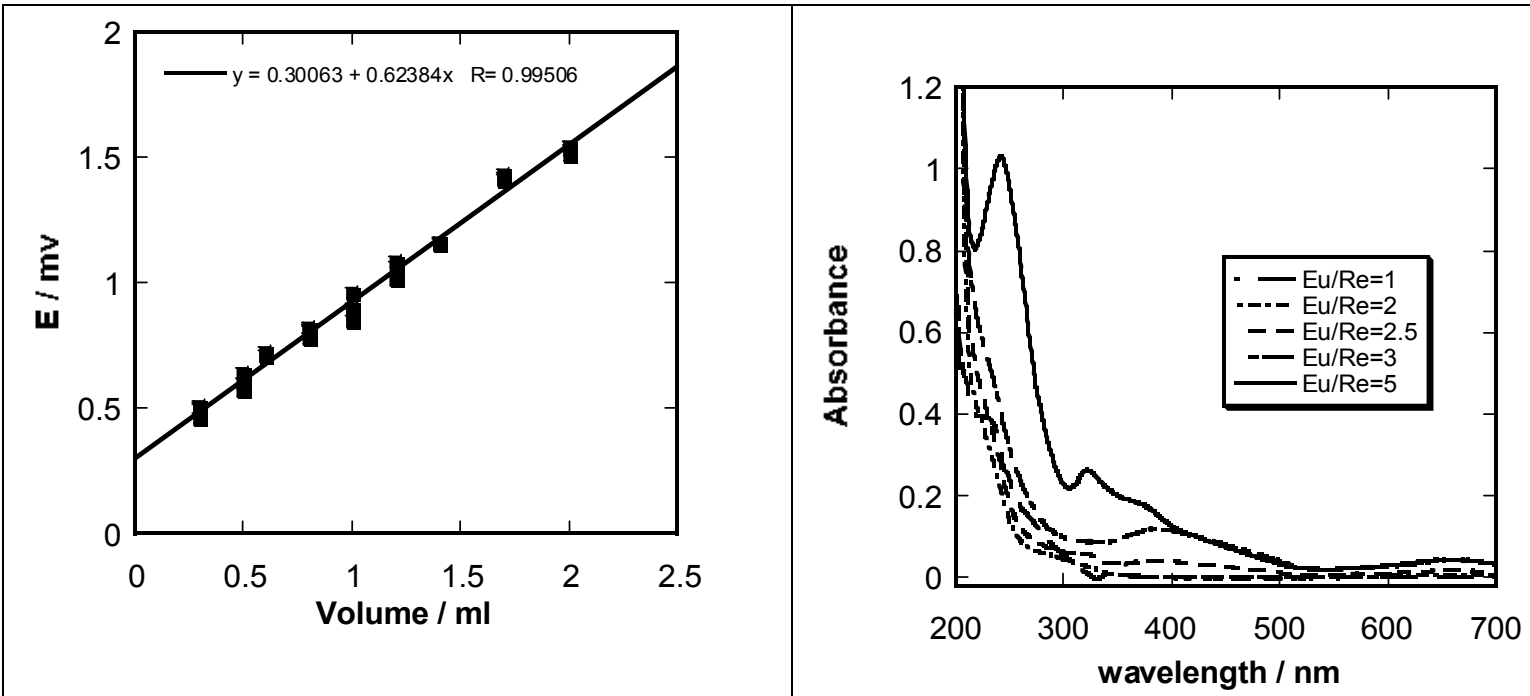

Figure S-1. Calibration curve for hydrogen evolution.

Figure S-2. Spectrophotometric titrations of MTO with $\mathrm{Eu}^{2+}$. Conditions: $[\mathrm{MTO}]=0.2 \mathrm{mM} ;\left[\mathrm{H}^{+}\right]=0.6$ $\mathrm{M}, 1-\mathrm{cm}$ cell at $25^{\circ} \mathrm{C}$.

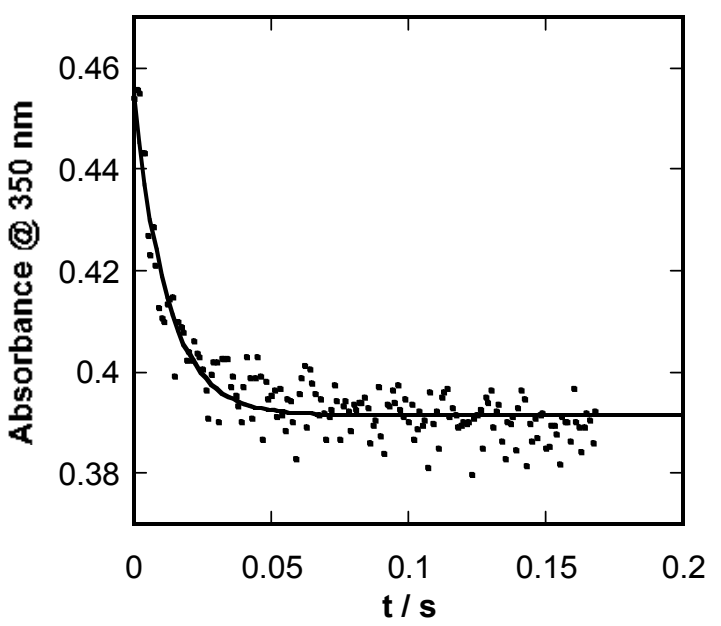

Figure S-3. Absorbance change showing that $\mathrm{Eu}^{2+}$ is rapidly oxidized by MTO. Conditions: $3.09 \mathrm{mM}$ MTO, $0.4 \mathrm{mM} \mathrm{Eu}^{2+}$ in $0.6 \mathrm{M} \mathrm{H}^{+}$at $25{ }^{\circ} \mathrm{C}$. The plot is fitted by pseudo-first-order kinetics with $\mathrm{k}_{\psi} 83.0 \mathrm{~s}^{-1}$.

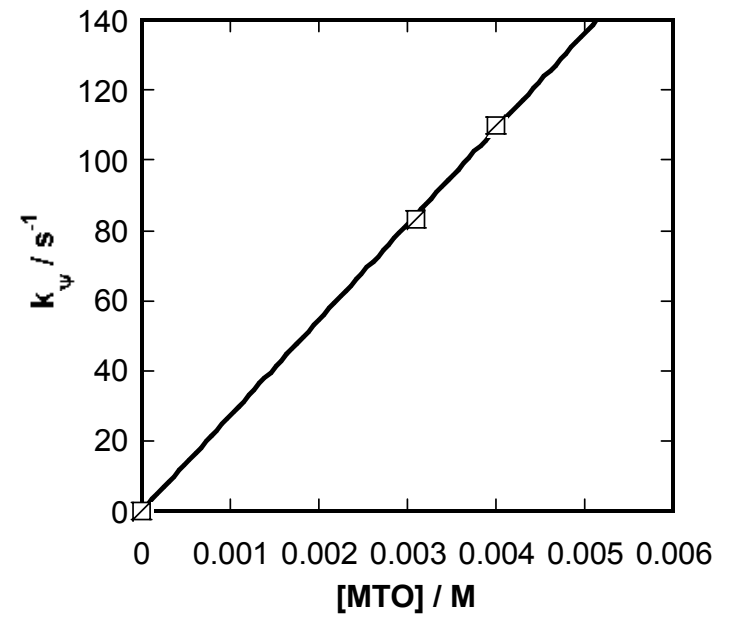

Figure S-4. Plot of $\mathrm{k}_{\psi}$ against the concentration of MTO for the $k_{1}$ reaction. The plot is fitted by a linear equation and yields $k_{1}=2.7 \times 10^{4} \mathrm{~L} \mathrm{~mol}^{-1} \mathrm{~s}^{-1}$ at 25 ${ }^{\circ} \mathrm{C}$. 


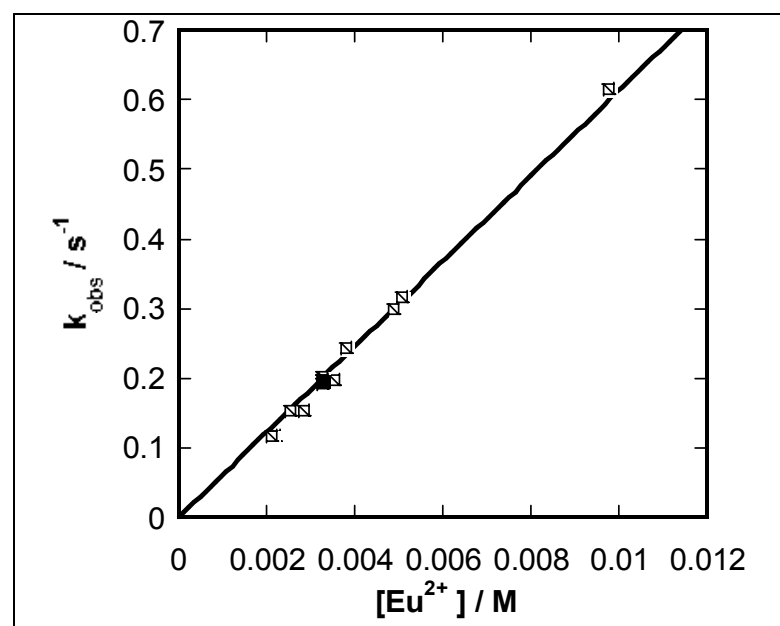

Figure S-5. Plot of $\mathrm{k}_{\mathrm{obs}}$ for eq 7 against the concentration of $\mathrm{Eu}^{2+}$, conditions: [MTO] $=0.2 \mathrm{mM}$, $\left[\mathrm{H}^{+}\right]=0.6 \mathrm{M}(\square),\left[\mathrm{H}^{+}\right]=0.2 \mathrm{M}(\mathbf{\square})$ at $25^{\circ} \mathrm{C}$.

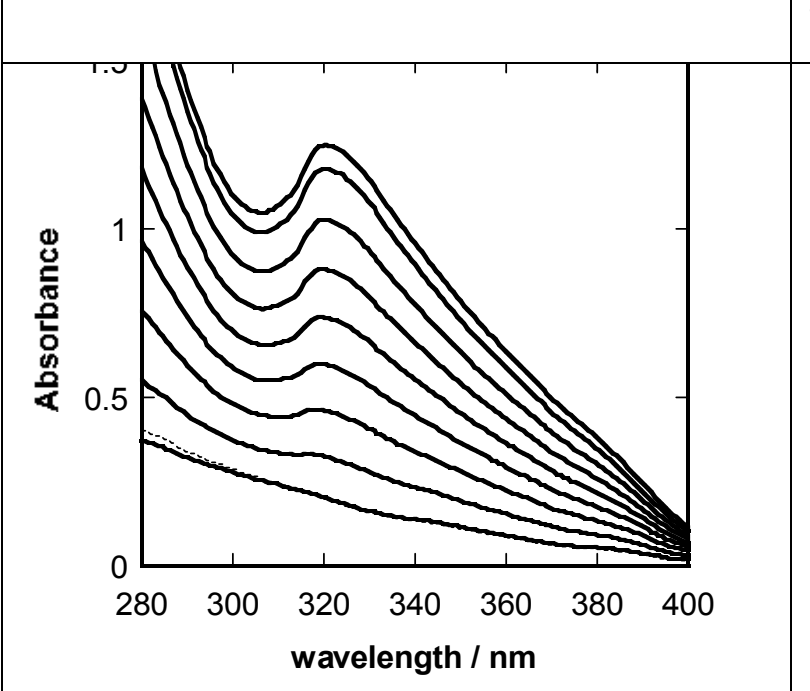

Figure S-7. Repetitive scans of UV-vis spectra representing the decrease of $\mathrm{Eu}^{2+}$ for perchlorate reduction at $1 \mathrm{~s}$ intervals. Condition: $\left[\mathrm{Eu}^{2+}\right]=1.55$ $\mathrm{mM},[\mathrm{MTO}]=0.1 \mathrm{mM} ;\left[\mathrm{ClO}_{4}^{-}\right]=0.05 \mathrm{M}$ and $\left[\mathrm{H}^{+}\right]=$ $0.55 \mathrm{M}$ with $\mu=0.8 \mathrm{M}$ at $25^{\circ} \mathrm{C}$.

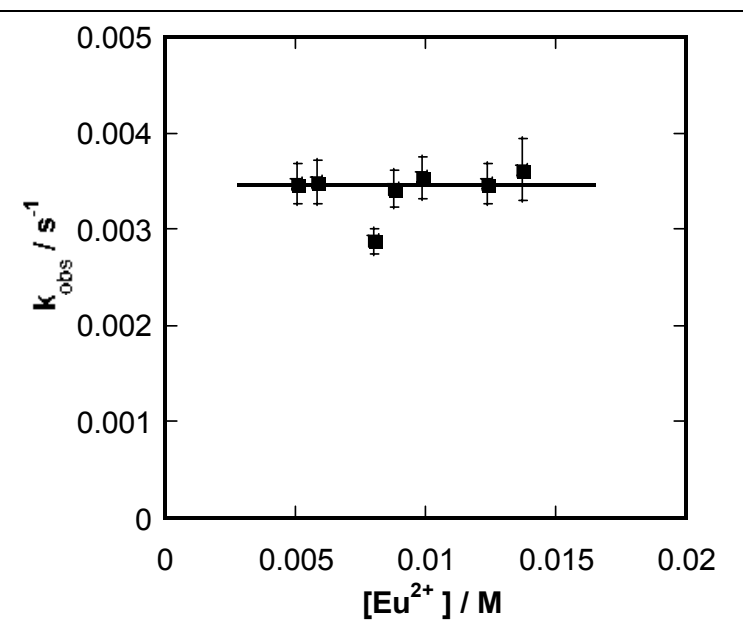

Figure S-6. Showing that the pseudo-first-order rate constant $\mathrm{k}_{\mathrm{obs}}$ remains independent of the $\mathrm{Eu}^{2+}$ concentration. Conditions: $[\mathrm{MTO}]=0.32 \mathrm{mM} ;[\mathrm{H}+]$ $=0.22 \mathrm{M}, \mu=0.3 \mathrm{M}$ and $\mathrm{Eu}^{2+}$ is the limiting reagent at $25^{\circ} \mathrm{C}$.

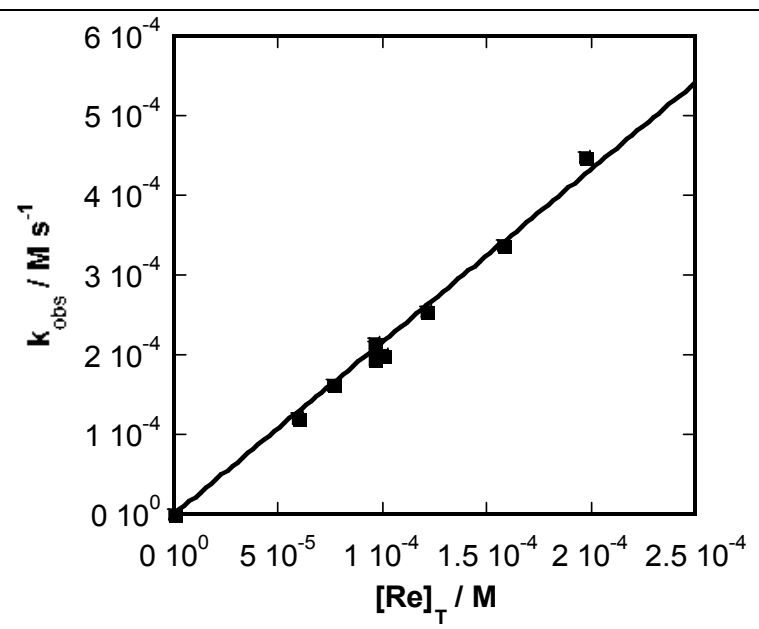

Figure S-8. Plot showing the linear variation of the pseudo-zero-order rate constant $\mathrm{k}_{\mathrm{obs}}$ with the concentration of $[\mathrm{Re}]_{\mathrm{T}}$. Condition: $\left[\mathrm{Eu}^{2+}\right]=1.23 \sim$ $2.00 \mathrm{mM},[\mathrm{MTO}]=0 \sim 0.2 \mathrm{mM} ;\left[\mathrm{ClO}_{4}{ }^{-}\right]=0.05 \mathrm{mM}$ and $\left[\mathrm{H}^{+}\right]=0.55 \mathrm{M}$ with $\mu=0.8 \mathrm{M}$ at $25^{\circ} \mathrm{C}$. 


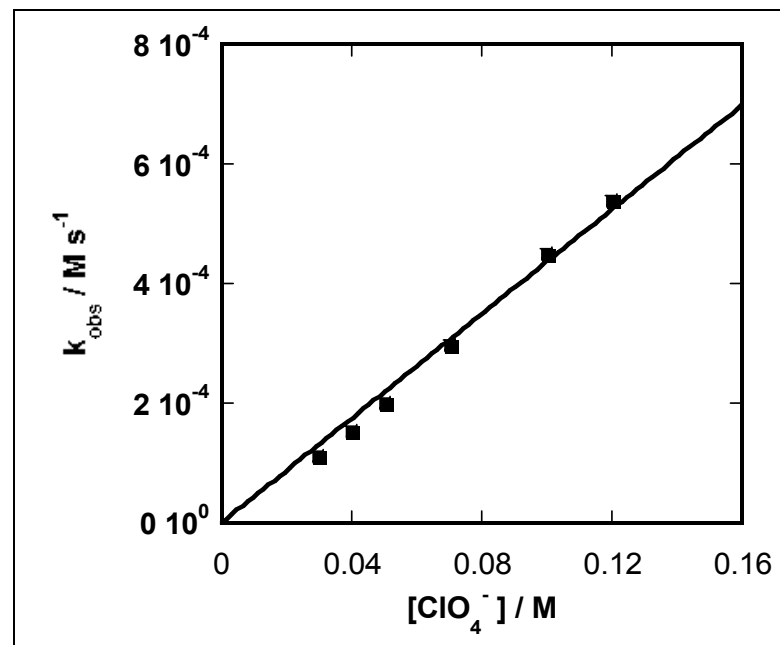

Figure S-9. Plot showing the first-order dependence upon $\left[\mathrm{ClO}_{4}^{-}\right]$for perchlorate reduction. Condition: $\left[\mathrm{Eu}^{2+}\right]=1.55 \mathrm{mM},[\mathrm{MTO}]=0.1 \mathrm{mM} ;\left[\mathrm{ClO}_{4}^{-}\right]=0.03$ $\sim 0.12 \mathrm{mM}$ and $\left[\mathrm{H}^{+}\right]=0.55 \mathrm{M}$ with $\mu=0.8 \mathrm{M}$ at 25 ${ }^{\circ} \mathrm{C}$.

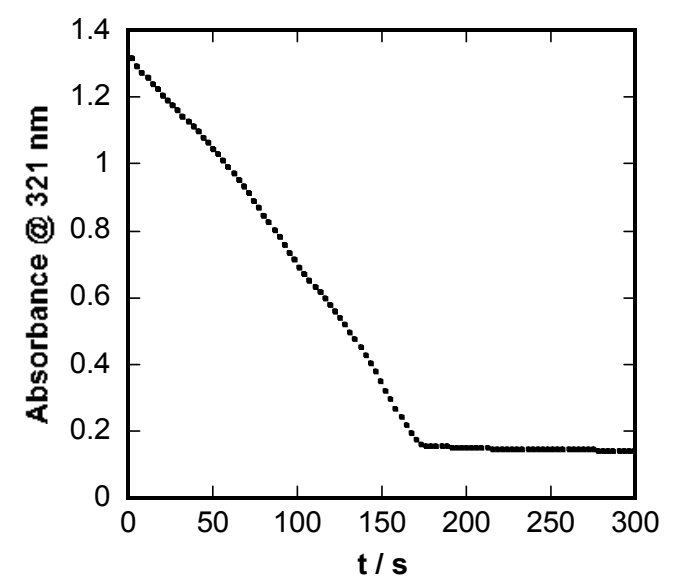

Figure S-11. Effect of the order of addition on perchlorate reduction with $2.92 \mathrm{mmol} \mathrm{L}^{-1} \mathrm{Eu}^{2+}, 0.20$ mmol L $\mathrm{MeReO}_{3}$, and $0.167 \mathrm{~mol} \mathrm{~L}^{-1} \mathrm{ClO}_{4}^{-}$(added last) at $0.50 \mathrm{~mol} \mathrm{~L}^{-1} \mathrm{H}^{+}, \mu=0.80 \mathrm{~mol} \mathrm{~L}^{-1}$ at $25^{\circ} \mathrm{C}$.

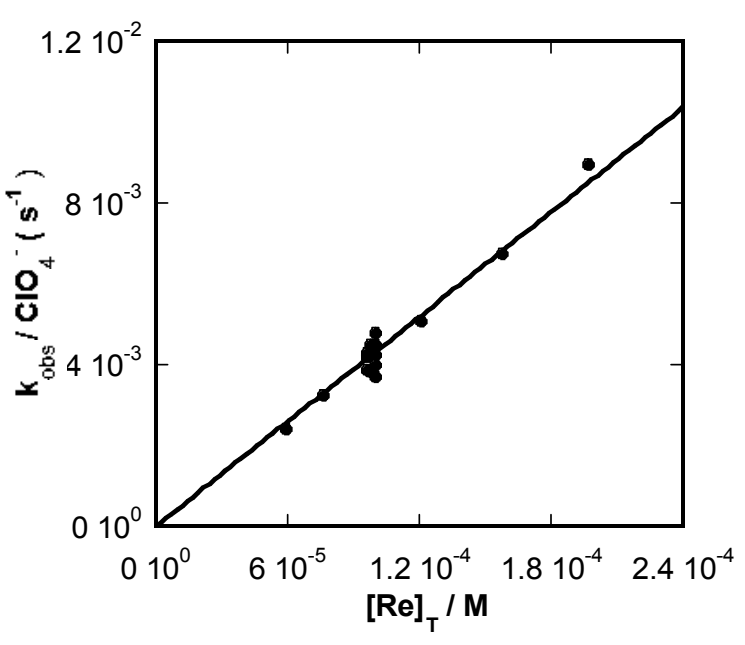

Figure S-10. Variations of $\mathrm{k}_{\mathrm{obs}} /\left[\mathrm{ClO}_{4}^{-}\right]$with $[\mathrm{Re}]_{\mathrm{T}}$ for perchlorate ion reduction.

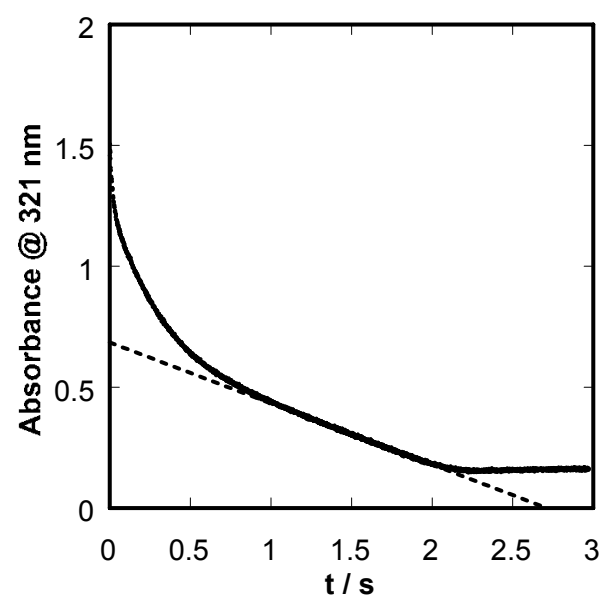

Figure S-12. Deliberate introduction of a trace of $\mathrm{O}_{2}$ greatly exaggerates the curvature during the MTOcatalyzed reduction of $\mathrm{ClO}_{4}^{-}$by $\mathrm{Eu}^{2+}$. Compare Figure 12 in the ms. 